\title{
Inhibitory Effects of Antifungal Drugs on Biofilm Producing Aspergillus spp. Recovered from Drinking Water System
}

\author{
Nazir NUA $^{1 *}$, Siddique $A^{2}$, Khan $\mathrm{MN}^{1}$ and Ishaque \\ $\mathbf{M}^{1}$ \\ ${ }^{1}$ University of Agriculture, Faisalabad, Pakistan \\ ${ }^{2}$ Atta -ur- Rahman School of Applied Biosciences, NUST, \\ Islamabad, Pakistan \\ *Corresponding author: Noor ul Ain Nazir, University \\ of Agriculture, Faisalabad, Pakistan
}

Received: April 20, 2020; Accepted: May 07, 2020; Published: May 14, 2020

\begin{abstract}
Aims: Biofilms formed in drinking water distribution systems serve as a continuous source of fungal infections. Biofilms are thick aggregates of adherent microorganisms including pathogenic species of fungi. Respiratory diseases and skin allergy reactions are caused by drinking water containing biofilm forming fungus and bacteria. One of the main causes of nosocomial infections and respiratory diseases in hospitals is due to the fungal biofilm formation in machines, catheters and other surgical instruments. There are some antifungal drugs which are used to control biofilm formation to minimize the infection rate.

Methodology and results: The present study was conducted to isolate and identify Aspergillus species which are the main fungal spp. responsible for the biofilm formation in drinking water and to check their antifungal susceptibility against antifungal drugs. The isolated fungal samples from drinking water were cultivated on Potato dextrose agar for the isolation of Aspergillus species. Isolated Aspergillus species were identified on the basis of cultural, morphological and microscopic examination. Then in-vitro ability of biofilm produced by isolated Aspergillus species was estimated using microtitre plate method and quantification by crystal violet assay. Antifungal susceptibility testing against isolated fungal spp. was done by antifungal drug Amphotericin B.

Results: From results, it is concluded that drinking water of labs, hospitals and common water chillers were more prevelant by Aspergillus species whereas water from reverse osmosis plants showed negative results. From microtitre plate method and crystal violet assay, it was concluded that Aspergillus spp. are Susceptible against Amphotericin B drug as compared to miconazole.
\end{abstract}

Keywords: Aspergillus spp; Biofilm; Drinking water; Disk diffusion method; Amphotericin B

\section{Introduction}

Quality of drinking water is of great importance for water consumers. One of the supreme concerns for the water consumers is contamination of drinking water with pathogenic microorganisms. These microbes include bacteria, viruses, protozoa and fungi, which are the causes of various water borne diseases and epidemics [1].

It is suggested that the occurrence of prominent fungal contaminations has been prevailing since the 1980s which causes increase in the number of immunocompromised patients such as patients of AIDS, tumor and individuals with chemotherapy treatment to get infections more easily. Furthermore, those microbes or fungus which thought to have low pathogenic potential have been found to bring about serious systemic ailment in immunocompromised hosts [2].

It is stated that fungal biofilms in a municipal water distribution system. Interpretation by scanning electron microscope proved that spores not hyphae were the primary source of possible propagates. The density level of filamentous fungi and yeasts showed that filamentous fungi were more common than yeasts in the formation of biofilm in drinking water. More established genera included Aspergillus, Alternaria, and Penicillium etc [3]. The biofilm developed inside the lining of water pipes mostly consists of opportunistic pathogens. Due to biofilm formation in water, different changes have been observed such as corrosion, water discoloration, and change in taste, water color and blockages of pipelines. The assessment of biofilm development inside of pipelines is challenging [4]. The estimation of fungi development in water, culturing techniques has been used. Moreover, high throughput sequencing techniques also used for the detection of biofilm development in water at advanced stages [5]. Fungus released its toxins in the water which causes various skin and respiratory diseases [6]. It is proposed that different factors affect the formation of biofilm. These factors includes hydraulic conditions, temperature and backwashing regime. Due to pathogenicity and enzymatic activities the chances for fungi to breakdown complex material and anthropogenic substances has increased. Fungus has bad effect on plants, invertebrates and microorganisms by producing mycotoxins. Taste and odor of water changed due to presence of fungi in it [7]. The biofilm infections are mostly relates to the skin and respiratory tract infections. In hospitals, patients who are immunocompromised are mostly at the edge of getting infections. Most of the hospitals 
infections occurred due to the biofilm of Aspergillus spp. whereas Aspergillus fumigatus is responsible for $90 \%$ infections [8]. Water is the basic entity on earth for life of every organism. They analyzed that drinking water in mineral bottles and tap water contaminated with different types of yeasts and filamentous fungi and found their relation with bacteria found in fecal samples. Different filter technique was used for yeasts and fungi. These were isolated on Sabouraud dextrose agar [9]. It is important to discuss the importance and applications of biofilm. Structurally biofilms are made up of microcolonies of different microbial cells (bacteria, yeasts, fungi, algae etc.) Which is $15 \%$ by volume and $85 \%$ constitutes the matrix material. The role of biofilm in microbial communities such as protection from environment, nutrient availability, acquisition of new genetic trait and penetration of antimicrobial agents was also studied [10].

In water environment, biofilm development has crucial significance. Microbes and fungus which live in biofilm are more resistant to antifungal agents and antibiotics. They are more likely to cause diseases. Biofilms mostly made bond of microbes to stones and some hard places [11].

This study was aimed to investigate the presence of Aspergillus $s p p$. in different drinking water sources, evaluate biofilm potential of isolated Aspergillus spp. and estimation of antifungal drugs (amphotericin B and miconazole) effect against biofilm producer Aspergillus spp.

\section{Materials and Methods}

\section{Sample collection}

A total 138 drinking water samples were collected from different areas of the Faisalabad city. 80/138 samples were collected from different drinking water sources such as water chillers, Hostels, Canteen water, Laboratories of University of Agriculture Faisalabad, 10/138/ samples from the reverse osmosis plants and 48/138 samples were collected from District Headquarters and Allied Hospital, Faisalabad. Water samples were collected in sterilized plastic bottles and transferred to the laboratory of Microbiology, University of Agriculture Faisalabad. Samples were preserved at $4^{\circ} \mathrm{C}$ for further processing.

\section{Isolation and Identification of Aspergillus spp}

For isolation of Aspergillus, water samples were spread on Potato Dextrose Agar (PDA) plates with the help of spreader. Then plates were incubated at $37^{\circ} \mathrm{C}$ for 5 days in incubator. After 5 days, inoculated plates were checked for fungal growth. For identification, fungal growth appeared on the Potato Dextrose Agar plates were analysed by microscopic and macroscopic examination. For the microscopic examination, slide culture technique was used while macroscopic examination was done on the basis of morphology of fungal growth.

\section{Spore suspension}

Spores of each isolate were harvested from 7 days old pure culture in Malt Extract Agar (MEA) by swirling 2mL of saline solution ( $0.85 \%$ ) into plates. The spore suspension was suspended and vortexed before quantification. The suspensions were standardized by dilution with saline solution. Final concentrations of $10^{5} \mathrm{spores} / \mathrm{ml}$ were taken. Spores were counted by using a Neubauer counter chamber.

\section{Biofilm development}

Two isolated colonies were taken from the petri plates of the desired culture with sterilized loop and inoculated on Potato dextrose agar. Cultures were incubated overnight at $37^{\circ} \mathrm{C} .100 \mu \mathrm{l}$ of culture was taken and added in the fresh PDA broth to make the volume of $10 \mathrm{ml}$ with dilution 1:10. Each diluted culture of $100 \mu \mathrm{l}$ of both Aspergillus spp. taken added in the microtitre plates. Then, $50 \mu \mathrm{l}$ of the distilled water and $100 \mu \mathrm{l}$ of Potato Dextrose Agar (PDA) added in the microtitre plate respectively. Two microtitre plates contain suspension of Aspergillus fumigatus and Aspergillus flavus each were incubated for 5 days at $37^{\circ} \mathrm{C}$. Biofilm formation was clearly seen after 5 days.

\section{Microscopy examination}

Biofilm formation was visualized under compound microscope and stereomicroscope. The magnification of stereo microscope and compound microscope used were 4.5X and 1000X respectively. After microscopic examination, growth medium was discarded and micro titer plates were washed twice with $200 \mu \mathrm{l}$ of $0.85 \%$ normal saline to remove loosely attached fungus residues. Plates were air dried at $30^{\circ} \mathrm{C}$ for 30 minutes.

\section{Crystal Violet Assay}

Biofilm containing wells in microtitre plate were stained with 200 $\mu \mathrm{l}$ of $0.1 \%$ crystal violet for 10 minutes at room temperature. Excess dye was removed by washing each well three times with $0.85 \%$ normal saline. Then, $200 \mu \mathrm{l}$ of $33 \%(\mathrm{v} / \mathrm{v})$ acetic acid added in all wells. 100 $\mu \mathrm{l}$ of acetic acid from all wells was transferred to new plate. Optical Density (OD) of micro titer was measured by microtitre plate reader at wave length of $450 \mathrm{~nm}$.

\section{Antifungal susceptibility testing by disk diffusion method}

Evaluation of antifungal susceptibility against antifungal drugs was done by Disk diffusion method. Disks of same size were taken, dip them in the Amphotericin B drug solution and placed in the plates containing Aspergillus fumigatus and Aspergillus flavus growth. Results were seen after 2 days.

\section{Results}

\section{Sample collection}

Out of 138 samples, 87 samples were positive for Aspergillus species, of which 35 samples were Aspergillus flavus and 52 samples were Aspergillus fumigatus.

\section{Identification of Fungi}

Fungi were identified on the basis microscopic and macroscopic examinations such as colony morphology, spore structure and conidia structure. Initially, Aspergillus species were identified on the basis of morphological characteristics such as colony texture, colony appearance, and color and conidia structure. Color of the fungal colonies was blackish and brownish whereas slide culture technique showed the structure of fruiting bodies of fungus. The images were visualized under light and compound microscope at 100 and $1000 \mathrm{X}$ magnification respectively. On the basis of microscopic and macroscopic examination, the fungus species were identified as Aspergillus fumigatus and Aspergillus flavus. Figure 1 and 2 shows the macroscopic examination, growth of Aspergillus spp. on Potato dextrose agar and Malt extract agar.

As shown in Figure 1, Colony morphology of Aspergillus fumigatus was first brownish in color and then change to blackish 


Table:
\begin{tabular}{|c|c|c|c|}
\hline Sr No & Water Source & No. of water samples & Aspergillus spp in samples. \\
\hline 1 & University research Labs, canteens & 50 & $\begin{array}{c}\text { Type of spp. } \\
20=A . F u m i g a t u s . ~ \\
15=A . f l a v u s\end{array}$ \\
\hline 2 & Public water chillers & 30 & $35=$ A.Fumigatus. \\
$10=$ A.flavus & $13=$ A.Fumigatus. \\
\hline 3 & Hospitals tap water & 48 & 20 \\
\hline 4 & Reverse osmosis plants & 10 & Nonus \\
\hline
\end{tabular}

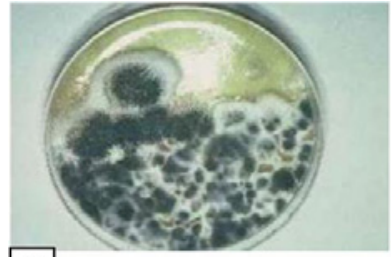

$\mathrm{A} \mid$

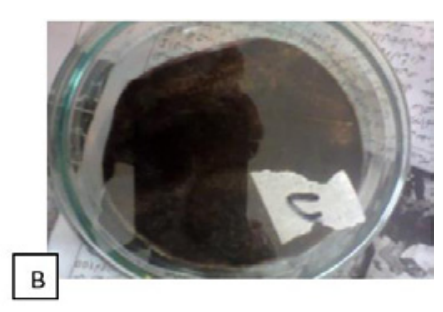

Figure 1: Macroscopic examination of Aspergillus fumigatus.

A: Blackish and brownish growth of Aspergillus fumigatus on Malt extract agar.

B: Blackish brownish growth of Aspergillus fumigatus on Potato dextrose agar
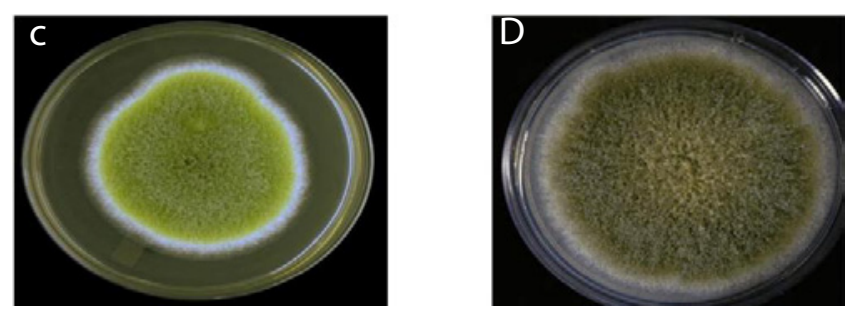

Figure 2: Macroscopic identification of Aspergillus flavus.

C: Aspergillus flavus growth on Malt extract agar.

D: Aspergillus flavus growth on Potato dextrose agar.

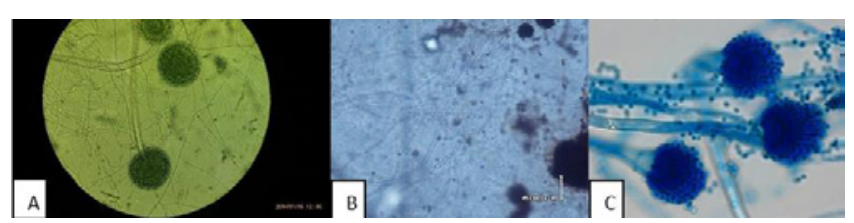

Figure 3: Microscopic examination of Aspergillus fumigatus.

A: Aspergillus fumigatus under light microscope at 100X magnification.

B: Aspergillus fumigatus under compound microscope at 250X magnification.

C: Aspergillus fumigatus under compound microscope at $1000 \mathrm{X}$ magnification.

color. Colony was granular to cottony velvety and powdery usually white to black in color.

As shown in Figure 2, the morphology of Aspergillus flavus is whitish greenish in color. The appearance of Aspergillus flavus is wooly and velvety. And later it becomes brownish whitish in color on PDA.

As shown (Figure 3) spores of Aspergillus fumigatus are light in color and blackish greenish in color. The hyphae are septate with rough colorless conidia. Green to grey conidia $(2-3 \mu \mathrm{m})$. The conidia structure is rough, smooth and slightly rough form long chains. Philades are flask shaped closely spaced in chains. Philades they tend to found on the upper vesicle extend parallel to conidiophores.

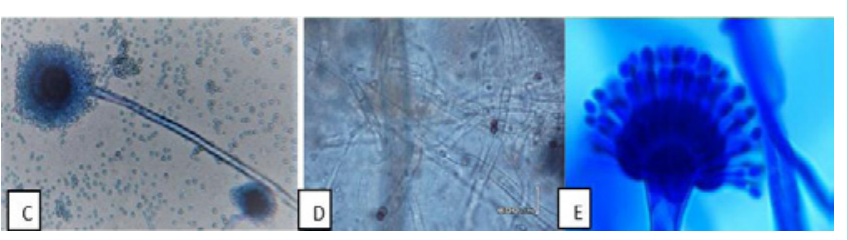

Figure 4: Microscopic examinations of Aspergillus flavus.

C. Aspergillus flavus under light microscope at 100X magnification.

D. Aspergillus flavus under compound microscope at 250X magnification.

E. Aspergillus flavus under compound microscope at 1000X magnification.

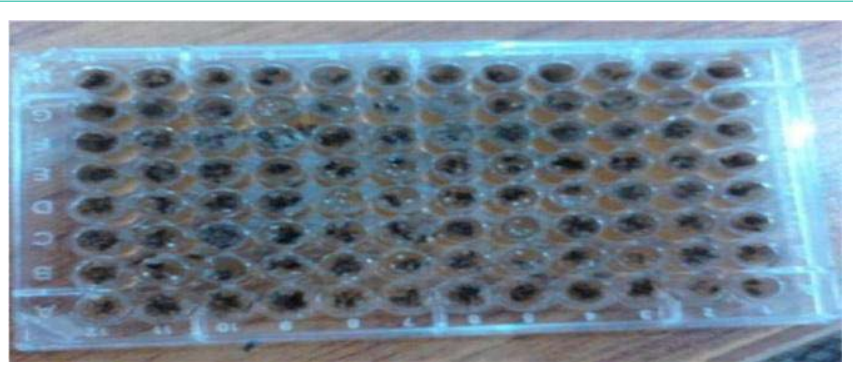

Figure 5: Biofilm formation in micro titer plate.

As shown in (Figure 4), conidiophores are rough colorless. Hyphae are septate and hyaline. Conidia are thin wall; shape is from spherical to elliptical. Conidia arise in chains. Philades radiates from vesicles in all directions whereas septate hyphae with rough colorless conidia.

Biofilm formation was seen after three and five days. Mature biofilm formation visualized clearly after 5 days of incubation as shown in Figure 5.

\section{Biofilm formation assay}

Biofilm formation was seen after three and five days. Mature biofilm formation visualized clearly after 5 days of incubation

In Figure 6, Figure A: the well showed the control well of microtitre plate. In Figure B: showed formation of biofilm by Aspergillus fumigatus seen under 4.5X magnification stereomicroscope. Figure C: showed the image of mature biofilm formation by Aspergillus fumigatus seen under $4.5 \mathrm{X}$ magnification stereomicroscope

In Figure 7, Figure D: showed the control well of microtitre plate. Figure E: well showed the formation of biofilm by Aspergillus flavus after 3 days seen under 4.5X magnification, stereomicroscope. Figure F: showed the mature biofilm formation by Aspergillus flavus at 4.5 $\mathrm{X}$ magnification under stereomicroscope. Clearly difference was seen between control wells and wells containing growth.

\section{Crystal violet assay}

The results of Crystal Violet assay interpreted through Graphs 


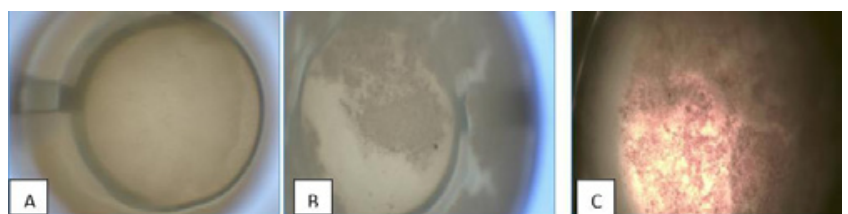

Figure 6: Biofilm formation by Aspergillus fumigatus

A: Control well of Microtitre plate.

B: Biofilm formation by Aspergillus fumigatus after 3 days at $4.5 \times$ under stereomicroscope.

C: Biofilm formation by Aspergillus fumigatus after 5 days at $4.5 \mathrm{X}$ magnification under stereomicroscope.

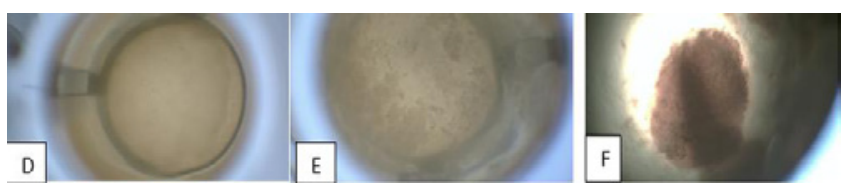

Figure 7: Biofilm formation by Aspergillus flavus.

D: Control well of Microtitre plate.

E: Biofilm formation by Aspergillus flavus under stereomicroscope at $4.5 \mathrm{X}$ magnification after 3 days.

F: Biofilm formation by Aspergillus flavus under stereomicroscope at $4.5 \mathrm{X}$ magnification after 5 days.

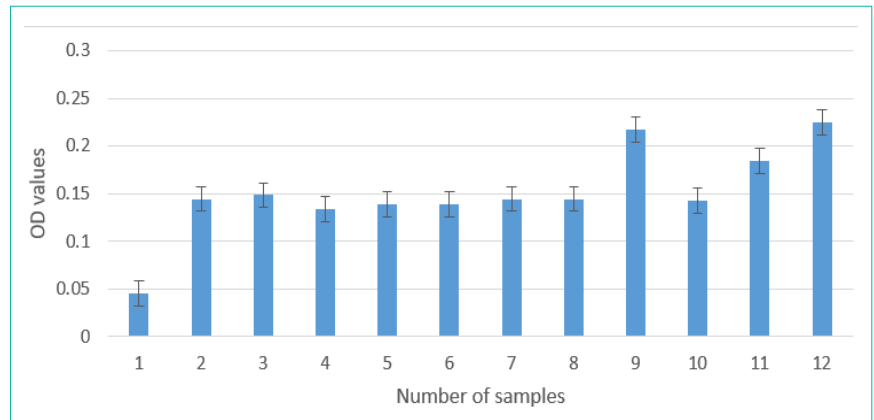

Graph 1: Geometric mean of OD values of Aspergillus flavus.

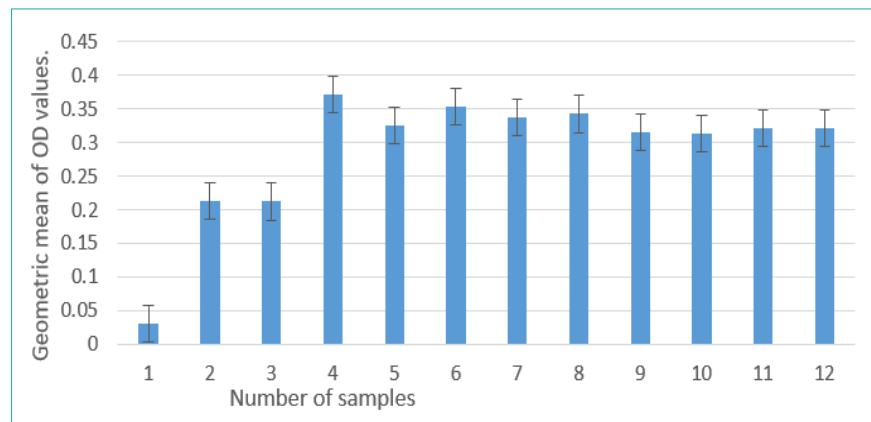

Graph 2: Geometric mean of OD values of Aspergillus fumigatus.

and Tables shown below. The Crystal violet assay OD values of biofilm formation by Aspergillus fumigatus was found to be numerically, statistically significantly higher among all results as compared to Aspergillus flavus. In Graphs 1, 2, the results showed that Aspergillus fumigatus capacity to form biofilm is higher than Aspergillus flavus.

Maximum Biofilm formation was observed in graphs by interpreting the results of crystal violet assay. The Biofilm formation values of Aspergillus fumigatus was found to be numerically, statistically significantly higher among all results. Biofilm of A. flavus showed low values of biofilm formation as compared to A. fumigatus biofilm. The values of Aspergillus flavus is between 0.04 to 0.23 whereas the values of Aspergillus fumigatus lie between 0.05 to 0.37 .

\section{Disk Diffusion method for Antifungal susceptibility testing}

Disk diffusion method was used for checking the antifungal susceptibility of amphotericin B and miconazole against Aspergillus spp. As shown in Figure 7, Zone of inhibition for miconazole and amphotericin B were $7 \mathrm{~mm}$ and $14 \mathrm{~mm}$ respectively for $A$. fumigatus whereas zone of inhibition for miconazole and amphotericin B were $6 \mathrm{~mm}$ and $13 \mathrm{~mm}$ respectively for A. flavus. It was found that Amphotericin B is more effective drug as compared to miconazole against both Aspergillus spp.

\section{Discussion}

In recent research, the drinking water samples were tested for the detection of biofilm formation. Two species were prominent in these samples: Aspergillus fumigatus and flavus. After making standard spore suspension, biofilm formation procedure was initiated Biofilm formation involved five steps attachment, adsorption, microcolony formation ( $1^{\text {st }}$ phase), microcolony formation ( $2^{\text {nd }}$ phase) and maturation development [12].

In formation of biofilm dispersal step is most important. The locality is also much important for the formation of biofilm. Fungal biofilm in water can pass through various treatments by different methods for solution of problems. Biofilm is actually the growth of various microbes and fungal which form a complex network. [13]. Aspergillus specie is most common for formation of biofilm. The subsequent disease, intrusive aspergillosis, is a cause of high mortality. The isolation techniques (spread plate, pour plate and membrane filtration) have been used for measurement of microorganisms in drinking water sources [14].

In recent research, biofilm development in microtitre plate was observed. Aspergillus fumigatus suspension along with potato dextrose agar and distilled water was poured in wells of one microtitre plate and Aspergillus flavus suspension in another microtitre plate and give incubation. After 5 days, mature biofilm formation was seen in plates. Mature biofilm in micro titer plate was examined under stereomicroscope at $4.5 \mathrm{X}$ magnification. The quantification of biofilm was performed with Crystal Violet assay (CV) in Elisa reader at $480 \mathrm{~nm}$.

From the results, it was concluded that Aspergillus fumigatus has more ability to form biofilm than Aspergillus flavus. The wells showed higher values has higher biofilm biomass whereas the wells showed lower values has less biomass.

The agar diffusion techniques (disk diffusion and E test usually utilized for antibacterial testing have additionally been connected to antifungal susceptibility testing). Amphotericin B (AMPH-B) had been the highest level for treatment of aspergillosis and was generally utilized at larger scale [15]. In Japan, new antifungals, for example, Iitraconazole (ITCZ), Voriconazole (VRCZ), Micafungin (MCFG), liposomal amphotericin B and caspofungin were familiar with the business in 1993, 2002, 2005, 2006 and 2012, individually. Disk diffusion method is more effectively respond to Amphotericin B and 
Voriconazole for the susceptibility testing of Aspergillus spp than any other technique [16].

From the results of Disk diffusion method, it is concluded that amphotericin B is more effective as compared to miconazole

\section{Conclusion}

From the above research, it is concluded that most of the respiratory diseases are occurring due to the contaminated drinking water and that contamination is due to biofilm. In recent research, Aspergillus $s p p$. is found in high amount in drinking water. It is concluded from the results, that Aspergillus fumigatus is more vulnerable to produce biofilm in drinking water as compared to Aspergillus flavus. It is also concluded that Antifungal drugs such as Amphotericin B is found to be strongly effective against both Aspergillus spp. So, it's a dire need to clean the sources of water such as chillers in universities, hospitals and canteens regularly by using antifungal drugs or sprays.

\section{References}

1. Economou CN, IA Vasiliadou, G Aggelis, S Pavlou and DV Vayenas. Modeling of oleaginous fungal biofilm developed on semi-solid media. Bioresource Technol. 2010; 102: 9697-9704.

2. Butcher J, G Ramage and C Williams. Development of an Aspergillus fumigatus model to determine the effectiveness of antifungal treatments in vivo. Antimicrob Agents Ch. 2001; 45: 2475-2479.

3. Hageskar G, N Lima and I Skaar. The study of fungi in drinking water. Mycol Res. 2009; 113: 165-172.

4. Lin W, Y Zhisheng, Z Hongxun and PT lan. Diversity and dynamics of microbial communities at each step of treatment plant for potable water generation. Wat Res. 2013; 52: 218-230.

5. Harding MW, LRM Lyriam, JH Ronald and EO Merle. Can filamentous fungi form biofilms? Trends in Microbiol. 2011; 17.

6. Siqueira VM, HBO Helena, S Cledir, RMP Russell, BG Norma and N Lima Filamentous fungi in drinking water, particularly in relation to biofilm formation. Int J Environ Res and Public Health. 2011; 8: 456-469.
7. Gomes IB, M Simoes and LC Simoes. An overview on the reactors to study drinking water biofilms. Wat Res. 2014; 62: 63-87.

8. Anaissie EJSLS, MC Dignani, RC Summerbell, JH Rex, TP Monson, T Spencer, M Kasai, A Francesconi and TJ Walsh. Pathogenic Aspergillus species recovered from a hospital water system. J Clin Infect Dis. 2002; 34: 780-789.

9. Okpako, EC, AN Osuagwu, AE Duke and VO Ntui. Prevalence and significance of fungi in sachet and borehole drinking water in Calabar, Nigeria. Afr J Microbiol. 2009; 3: 56-61.

10. Yamaguchi MU, RCP Rampazzo, SFY Ogatta, CV Nakamura, TU Nakamura and BPD Filho. Yeasts and filamentous fungi in bottled mineral water and tap water from municipal supplies. Braz Arch Biol Techn. 2007; 50: 1-9.

11. Liu R, Z Junge, Y Zhisheng, J DevRaj, Z Hongxun, L Wenfang and Y Min. Molecular analysis of long-term biofilm formation on PVC and cast iron surfaces in drinking water distribution system. J Environ Sci. 2013; 26: 865874.

12. Goncalves $A B, M P R R$ and $L$ Nelson. Survey and significance of filamentous fungi from tap water. Int J Hyg Environ Health. 2005; 209: 257-264.

13. Tamiya H, O Eri, K Kazuyo, Y Maki, T Takahito, W Akira, Y Takashi and K Katsuhiko. Secondary metabolite profiles and antifungal drug susceptibility of Aspergillus fumigatus and closely related species, Aspergillus lentulus, Aspergillus udagawae and Aspergillus viridinutans. J Infect Ch. 2015; 21: 385-391.

14. Nourmoradi H, MN, CRS and HM. Ultraviolet irradiation: An effective inactivation method of Aspergillus spp. in water for the control of waterborne nosocomial aspergillosis. Wat Res. 2012; 46: 5935-5940.

15. Mowat E, John B, Sue L, Craig W and Gordon R. Development of a simple model for studying the effects of antifungal agents on multicellular communities of Aspergillus fumigatus. J Med Microbiol. 2007; 56: 1205-1212.

16. Extremina CI, L Costa, Al Aguiar, L Peixe and AP Fonsec. Optimization of processing conditions for the quantification of enterococci biofilms using microtitre-plates. J Microbiol Meth. 2011; 84: 167-173. 